\title{
Bacteroides dorei dominates gut microbiome prior to autoimmunity in Finnish children at high risk for type 1 diabetes
}

\section{Austin G. Davis-Richardson ${ }^{1}$, Alexandria N. Ardissone ${ }^{1}$, Raquel Dias ${ }^{1}$, Ville Simell ${ }^{2}$, Michael T. Leonard', Kaisa M. Kemppainen' ${ }^{1}$ Jennifer C. Drew' ${ }^{1}$, Desmond Schatz ${ }^{3}$, Mark A. Atkinson ${ }^{4}$, Bryan Kolaczkowski ${ }^{1}$, Jorma Ilonen ${ }^{5,6}$, Mikael Knip $^{7,8,9}$, Jorma Toppari ${ }^{2}$, Noora Nurminen ${ }^{10}$, Heikki Hyöty ${ }^{10}$, Riitta Veijola ${ }^{11}$, Tuula Simell ${ }^{2}$, Juha Mykkänen ${ }^{2}$, Olli Simell ${ }^{2}$ and Eric W. Triplett ${ }^{1 *}$}

\footnotetext{
'Department of Microbiology and Cell Science, Institute of Food and Agricultural Sciences, University of Florida, Gainesville, FL, USA

${ }^{2}$ Department of Pediatrics, Turku University Hospital and University of Turku, Turku, Finland

${ }^{3}$ Department of Pediatrics, University of Florida, Gainesville, FL, USA

${ }^{4}$ Department of Pathology, Immunology and Laboratory Medicine, University of Florida, Gainesville, FL, USA

${ }^{5}$ Department of Clinical Microbiology, University of Eastern Finland, Kuopio, Finland

${ }^{6}$ Immunogenetics Laboratory, University of Turku, Turku, Finland

${ }^{7}$ Department of Pediatrics, Children's Hospital, University of Helsinki and Helsinki University Central Hospital, Helsinki, Finland

${ }^{8}$ Diabetes and Obesity Research Program, University of Helsinki, Helsinki, Finland

${ }^{9}$ Department of Pediatrics, Tampere University Hospital, Tampere, Finland

${ }^{10}$ School of Medicine, University of Tampere, Tampere, Finland

"Department of Pediatrics, University of Oulu, and Oulu University Hospital, Oulu, Finland
}

\section{Edited by:}

John R. Battista, Louisiana State

University and Agricultural and

Mechanical College, USA

Reviewed by:

Suleyman Yildirim, Istanbu Medipol University, Turkey

Michael S. Allen, University of North

Texas Health Science Center, USA

*Correspondence:

Eric W. Triplett, Department of Microbiology and Cell Science, Institute of Food and Agricultural

Sciences, University of Florida, 1355

Museum Rd., PO Box 110700,

Gainesville, FL 32611-0700, USA

e-mail: ewt@ufl.edu
The incidence of the autoimmune disease, type 1 diabetes (T1D), has increased dramatically over the last half century in many developed countries and is particularly high in Finland and other Nordic countries. Along with genetic predisposition, environmental factors are thought to play a critical role in this increase. As with other autoimmune diseases, the gut microbiome is thought to play a potential role in controlling progression to T1D in children with high genetic risk, but we know little about how the gut microbiome develops in children with high genetic risk for T1D. In this study, the early development of the gut microbiomes of 76 children at high genetic risk for T1D was determined using high-throughput $16 \mathrm{~S}$ rRNA gene sequencing. Stool samples from children born in the same hospital in Turku, Finland were collected at monthly intervals beginning at 4-6 months after birth until 2.2 years of age. Of those 76 children, 29 seroconverted to T1D-related autoimmunity (cases) including 22 who later developed T1D, the remaining 47 subjects remained healthy (controls). While several significant compositional differences in low abundant species prior to seroconversion were found, one highly abundant group composed of two closely related species, Bacteroides dorei and Bacteroides vulgatus, was significantly higher in cases compared to controls prior to seroconversion. Metagenomic sequencing of samples high in the abundance of the $B$. dorei/vulgatus group before seroconversion, as well as longer $16 \mathrm{~S}$ rRNA sequencing identified this group as Bacteroides dorei. The abundance of $B$. dorei peaked at 7.6 months in cases, over 8 months prior to the appearance of the first islet autoantibody, suggesting that early changes in the microbiome may be useful for predicting T1D autoimmunity in genetically susceptible infants. The cause of increased $B$. dorei abundance in cases is not known but its timing appears to coincide with the introduction of solid food.

Keywords: Bacteroides dorei, Bacteroides vulgatus, type-1 diabetes, autoimmunity, microbiome

\section{INTRODUCTION}

Type 1 diabetes (T1D) is an autoimmune disorder typically developing within the first 5-6 years of life, beginning with inflammation of the pancreas, progression to autoimmunity and ultimately destruction of insulin-producing beta cells in the pancreas, resulting in dependence on daily exogenous insulin to control blood glucose levels. T1D is a complex disorder whose etiology is poorly understood, but it is widely believed that a combination of genetic and environmental factors contribute to increased T1D risk (Knip et al., 2005). Disease incidence rate varies dramatically by country with Finland having the highest known rate (Karvonen et al., 1993). While genetics explains 50\% of T1D risk (Atkinson et al., 
2014), the acceleration in this disease's incidence over the last 50 years in developed countries strongly suggests that T1D propensity is increasingly due to environmental factors (Karvonen et al., 1993).

Evidence from both rodent models and human studies suggests that the composition of the gut microbiome has a significant influence on the immune system development and may influence T1D risk. Feeding of antibiotics (Brugman et al., 2006; Schwartz et al., 2007) or a probiotic-like bacterial strain (Valladares et al., 2010) reduced autoimmune diabetes in disease-prone rodent models. Non-obese diabetic (NOD) mice raised in germ-free environments have an increased risk for diabetes (King and Sarvetnick, 2011) while exposure to bacterial antigens and infection decrease T1D risk (Bach, 2002). NOD mice that lack an adaptor for multiple innate immune receptors have an altered microbiome compared to control strains (Wen et al., 2008). In rats with diabetes, a culture-independent study showed increased proportions of Bacteroides and decreased Lactobacillus, compared to control rats (Roesch et al., 2009b). In humans, gut microbiome analysis of small cohorts of T1D autoimmune and control children showed significant bacterial taxa and functional differences (Brown et al., 2011; Giongo et al., 2011; de Goffau et al., 2013). More recently, a large case-control study from the BABYDIET study cohort (Schmid et al., 2004; Hummel et al., 2011) showed that microbial interaction networks were impaired in case samples, although no significant differences between the abundances of bacterial taxa were identified in cases versus controls (Endesfelder et al., 2014).

The evidence of a microbial role in the development of Type-1 diabetes lead to the formation of several studies on human cohorts including TEDDY (TEDDY Study Group, 2008), BABYDIET, and this study. These studies share several features including being retrospective, longitudinal across age, and controlling for genotype by selecting only subjects at moderate to high genetic risk. This study is the largest published study to date in terms of numbers of samples as well as subjects and is the only study where all subjects were from the same city and born in the same hospital thus controlling for many environmental factors which may confound studies of T1D and the microbiome. Results reported here from a large Finnish cohort support an association of a single species of bacteria, Bacteroides dorei, in the development of T1D autoimmunity in children at high risk for the disease. While this association does not establish a causative role in T1D development, observed changes in the gut microbiome occurred before development of the disease and could potentially be used as a biomarker for early screening.

\section{MATERIALS AND METHODS IRB APPROVAL}

Prior to commencing with this work, the University of Florida's Institutional Review Board assessed and approved this work as an exempt project with number 278-2010.

\section{COLLECTION OF STOOL SAMPLES}

The Finnish Type 1 Diabetes Prediction and Prevention Study (DIPP) began genetic screening of newborn infants from the general population in 1994 (Nejentsev et al., 1999). Newborns were screened for high-risk HLA-DR and HLA-DQ genotypes using a previously described method (Nejentsev et al., 1999). Monthly stool samples were collected by the subjects' parents at home and mailed to the DIPP Virus Laboratory for virology in Tampere, Finland, where they were stored at -20 to $-80^{\circ} \mathrm{C}$. In this study, 76 subjects were retroactively selected to create a cohort of agematched genotype-controlled subjects for the investigation of the microbiome as an environmental factor influencing risk of autoimmunity.

Subjects were born between 1996 and 2007 at the Turku University Hospital, Turku located in southwestern Finland (Table S1). A total of 947 stool samples were collected between 1996 and 2010 at monthly intervals when subjects were between 4-6 months old until to 2.2 years old (Table S2). Mode of delivery, duration of exclusive and total breast feeding, and antibiotics use were recorded (Tables S1, S4). As all samples were collected from within $80 \mathrm{~km}$ of the Turku University Hospital, the time from stool collection to frozen storage was likely not long. Previous work with Turku stool samples has shown minimal change to the microbial community if frozen within $24 \mathrm{~h}$ (Roesch et al., 2009a).

Detection of anti-islet autoimmunity was performed as described in Parikka et al. (2012). According to the DIPP data collected by 30 November 2011, the following groups of children with available stool samples were included in the analyses: cases $(n=29)$ who developed at least two persistent T1D-associated autoantibodies of whom 22 (75\%) progressed to T1D later during the follow-up and clinically unaffected healthy control children $(n=47)$ without any auto-antibodies (Table S1). These 47 subjects in the study did not express any islet autoantibodies during the course of the study and are from here referred to as controls.

Several known confounders of microbiome studies including breast feeding (total duration/any), duration exclusive breast feeding, mode of delivery, and antibiotic use (including amoxicillin and azithromycin, and 7 others) were compared between case and control groups via the chi-square test (Table S5, see Supplementary Methods) to ensure that these variables were balanced between cases and controls.

\section{DNA EXTRACTION, 16S rRNA AND METAGENOMIC SEOUENCING}

DNA was extracted from approximately 200-mg of stool sample using Qiagen AllPrep DNA/RNA/Protein Mini Kit (QIAGEN). Precautions were taken to avoid contamination, and blank amplification controls were processed adjacent to samples. DNA was quantified using a Nanodrop spectrophotometer (Thermo Scientific, Wilmington, DE). The primers used for 16S rRNA amplification and subsequent Illumina HiSeq sequencing were the V3 and V4 universal (Caporaso et al., 2011). For MiSeq sequencing, the $\mathrm{V} 3$ primer was replaced by the $314 \mathrm{~F}$ primer (Muyzer et al., 1996). Amplification 16S rRNA gene was performed as described previously (Fagen et al., 2012). PCR reactions were prepared with 25- $\mu$ l of GoTaq colorless Master Mix (Promega, Madison, WI), 10- $\mu \mathrm{M}$ of primers, 20-ng of sample DNA adjusted to $50-\mu l$ total volume with sterile, nuclease-free water. Samples were subjected to an initial denaturation temperature of $95^{\circ} \mathrm{C}$ for $3 \mathrm{~min}$, followed by 25 cycles of $95^{\circ} \mathrm{C}$ for $45 \mathrm{~s}$, $53^{\circ} \mathrm{C}$ for $30 \mathrm{~s}$, and $73^{\circ} \mathrm{C}$ for $90 \mathrm{~s}$, and a final elongation at $73^{\circ} \mathrm{C}$ for $10 \mathrm{~min}$. Amplified DNA was quantified fluorometrically with 
Qubit dsDNA High Sensitivity (Invitrogen, Life technologies Inc., Carlsbad, CA), and fragment size was verified on a gel. Neither DNA nor amplification was detected in blank control samples. Samples were randomly distributed across barcodes, lanes and sequencing runs to avoid any systematic bias. Equal masses of DNA from barcoded amplicons were merged and sequenced on the Illumina HiSeq 2000, and MiSeq platforms (Table S3).

\section{STO0L 16S rRNA SEQUENCING USING 2 × 100 bp ILLUMINA HISEO}

A total of 113 gigabases of nucleotide data were generated using three plates and seven lanes of Illumina HiSeq-2000 sequencing. Sequences were de-multiplexed based on the barcode sequence and reads with unmatched barcodes were discarded. Reads were trimmed for quality to remove bases with a high probability of error using the program Sickle (Joshi, 2013), and reads containing ambiguous nucleotides or that were shorter than 70 nucleotides after trimming were discarded. The $3^{\prime}$-most sequence in each pair was reverse-complemented so that all sequences were in the $5^{\prime}-3^{\prime}$ orientation. Removal of low quality bases as well as reads that contained ambiguous nucleotides resulted in an average of 375,313 $(S E=5107)$ reads per sample with an average length of $98 \pm$ 3 nucleotides. This length is adequate to assign reads to genus level (Liu et al., 2007). By discarding samples with fewer than 10,000 reads $(n=3)$ after trimming, 947 samples remained with a average of $357,581(S E=6400)$ reads per sample.

\section{MICROBIOME 16S rRNA GENE ANALYSIS}

To assign and quantify the Operational Taxonomic Units (OTUs) present in each stool sample, sequences were aligned to the GreenGenes 97\% representatives set version 13.5 (DeSantis et al., 2006) using the USEARCH program version 6.022 (Edgar, 2010), a component of QIIME (Caporaso et al., 2010), with 97\% minimum identity and 95\% minimum query coverage. In order to account for differences in sampling depth, read counts were subsampled using rarefaction with a sample size of 10,000 reads then divided by the total to calculate the percent relative abundance.

A total of 335 technical replicates were used to measure variation due to DNA extraction, amplification and differences in sequencing run and lane and sequencing technology (Table S3). Variance of relative abundance within technical replicates was found to sharply increase as relative abundance decreased (see Supplementary Methods). This trend was used to define a cutoff for minimum relative abundance below which accurate estimation of relative abundance is not possible. This cutoff was set at $1 \%$ relative abundance and taxa below the 99\% most-abundant taxa were excluded from the analysis due to their having high variance within technical replicates for DNA extraction, amplification and sequencing (Tables S6-S8). These filtered taxa made up a small portion of the total gut microbiome (Figures 1A,B). However, the total number of taxa including those below the cutoff was taken into account when performing the false discovery rate (FDR) correction.

\section{STATISTICAL COMPARISON OF CASE AND CONTROL MICROBIOMES}

The relative abundances of taxa at ranks Phylum, Genus, and Species were compared between all samples from all cases and controls using the non-parametric Mann-Whitney $U$-test (Tables S6-S8). To adjust for differences in per-subject sampling frequency, $p$-values were averaged over 100 bootstrap iterations with even per-subject sampling frequency and bootstrapped $p$ values were adjusted for FDR using the B-H method (Benjamini and Hochberg, 1995) using the total number of taxa including those that fell below 1\% average relative abundance. Significance was considered if the adjusted, bootstrapped $p$-value fell below alpha $=0.001$.

Logistic regression was used to model the interaction of age and relative abundance of each taxon with seroconversion as the response variable and model terms were tested for significant association with seroconversion. In this case, the model terms were the interaction between age and relative abundance. Significance of model terms was tested for using ANOVA. Model terms that fit significantly to seroconversion can be said to significantly vary between cases and controls. FDR-correction was applied to $p$-values using the same method as the Mann-Whitney $U$ test. The relative abundances of significant taxa were visualized across age using LOESS regression (Figure 2).

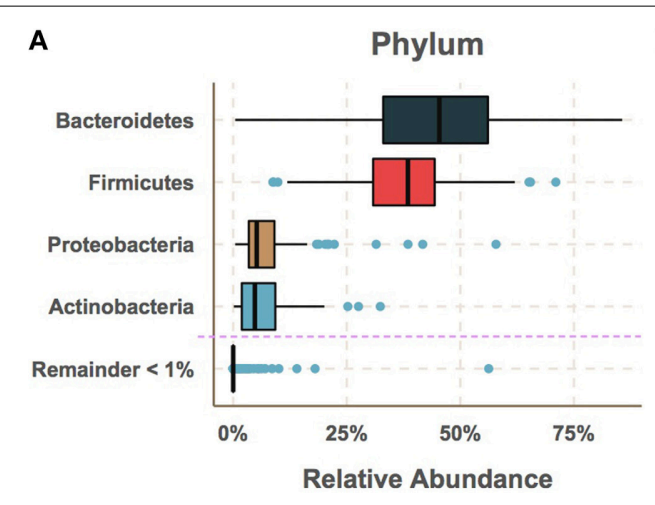

FIGURE 1 | The gut microbiome had low diversity and was dominated by a few taxa with median relative abundances greater than $1 \%$. (A) The most abundant phyla were Bacteroidetes and Firmicutes followed by Proteobacteria and Actinobacteria. (B) The most abundant genus was by far
B

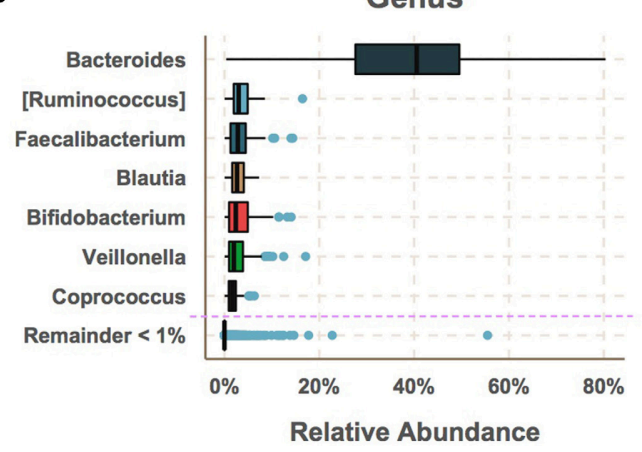

Bacteroides followed by Ruminococcus, Faecalibacterium, Blautia, Bifidobacterium Veillonella and Coprococcus. While a large number of species were detected, the majority of these had very low relative abundance. 

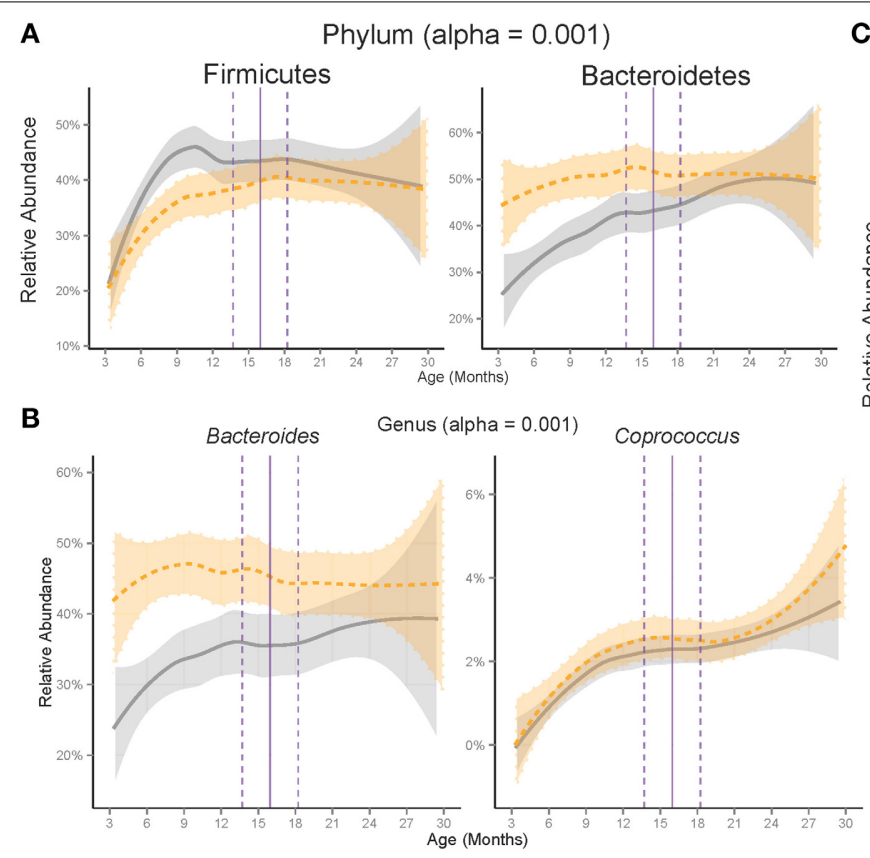

C Species (alpha $=0.001)$

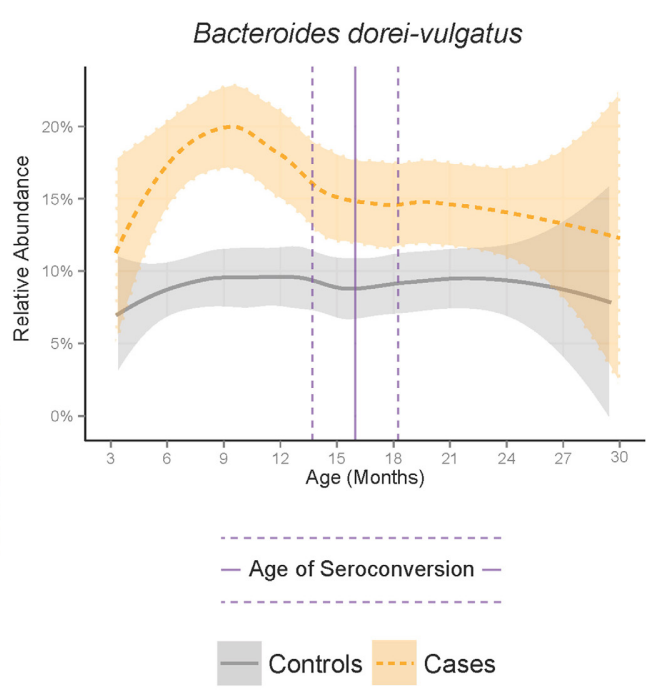

FIGURE 2 | Development of the microbiome differed between cases and controls at the (A) Phylum, (B) Genus and (C) Species ranks across age. Plotted are significant taxa with median relative abundance greater than $1 \%$.
Shaded region represents standard error of a LOESS smoothing regression. Vertical lines represent the median (solid) and standard error (dashed) age of appearance of first the autoantibody in cases.

\section{METAGENOMIC SEOUENCING OF SAMPLES ENRICHED FOR Bacteroides dorei}

DNA samples with a Bacteroides abundance above $47 \%$, as measured by $16 \mathrm{~S}$ rRNA analysis, were sequenced by the Pacific Bioscience RS-II platform with data assembled and analyzed as described previously (Leonard et al., 2014). Two of these four metagenomes used in this work were closed previously (Leonard et al., 2014, NCBI accession numbers CP007619 and CP008741). A third closed $B$. dorei genome is described here (NCBI accession number CP009057) which was obtained in the same manner from a healthy child, sample number 728. A fourth metagenome from a case subject, sample number 233 resulted in a partially assembled $B$. dorei genome. All four metagenomes were mined for $16 \mathrm{~S}$ and 23S rRNA using RNAmmer (Lagesen et al., 2007), as well as for a beta-glucosidase gene that has been reported to be present in B. dorei but lacking in B. vulgatus (Pedersen et al., 2013).

The 16S and 23S rRNA sequences mined from the metagenomic DNA were combined with sequences from Bacteroides species using Prevotella as an outgroup, aligned using MUSCLE and used to generate phylogenetic trees with PhyML using a GTR model (Figure 3A). These trees had nodes with higher (greater than 0.80 ) bootstrap support value and were capable of confidently separating $B$. dorei from $B$. vulgatus.

\section{IDENTIFICATION OF Bacteroides dorei AS THE MAIN COMPONENT OF THE Bacteroides dorei/vulgatus GROUP}

The length of the $2 \times 100 \mathrm{bp}$ Illumina HiSeq reads was insufficient to accurately distinguish $B$. dorei from $B$. vulgatus (Figure S1). Additional sequencing with overlapping $2 \times 350$ bp reads on the Illumina MiSeq platform to obtain assembled $450 \mathrm{bp}$ reads was conducted on a set of 93 samples with $>10 \%$ relative abundance of Bacteroides to improve taxonomic resolution of the $B$. dorei/B. vulgatus group. Reads were generated using a different forward primer (Muyzer et al., 1996), that targeted the 314-357 F region of the 16S rRNA gene. Overlapping MiSeq reads were assembled into single contigs using Pandaseq (Masella et al., 2012) and filtered to include only reads longer than $450 \mathrm{bp}$. Assembled MiSeq reads were trimmed to exactly $450 \mathrm{bp}$. A subset of 10,000 reads from each sample were clustered using de novo clustering at $99.6 \%$ identity by UCLUST (Edgar, 2010) to identify representative taxa. Representative sequences were combined with truncated 16S rRNA sequences from known Bacteroides species from GreenGenes and 16S rRNA sequences from the four PacBio metagenomes, aligned with MUSCLE (Edgar, 2004), and used to create a phylogenetic tree using PhyML with the GTR model (Guindon et al., 2005). A high bootstrap value (0.89) was measured in the branch separating $B$. dorei and $B$. vulgatus (Figure 3C). Representative sequences were then re-labeled as $B$. dorei and B. vulgatus depending on their placement in the phylogenetic tree and used as references to align the MiSeq reads to estimate relative abundance of the two groups and compared to the combined $B$. dorei and B. vuglatus relative abundance obtained from shorter reads.

Poisson linear regression was used to estimate the relative abundance of $B$. dorei and $B$. vulgatus based on the correlation between HiSeq relative abundance of the $B$. dorei/vulgatus group and MiSeq relative abundance of $B$. dorei and $B$. vulgatus separately (see Supplementary Methods). Samples were up-weighted based on relative abundance due to their measured increase in variance resulting in linear regressions with good fit $\left(R^{2}=0.94\right)$. 


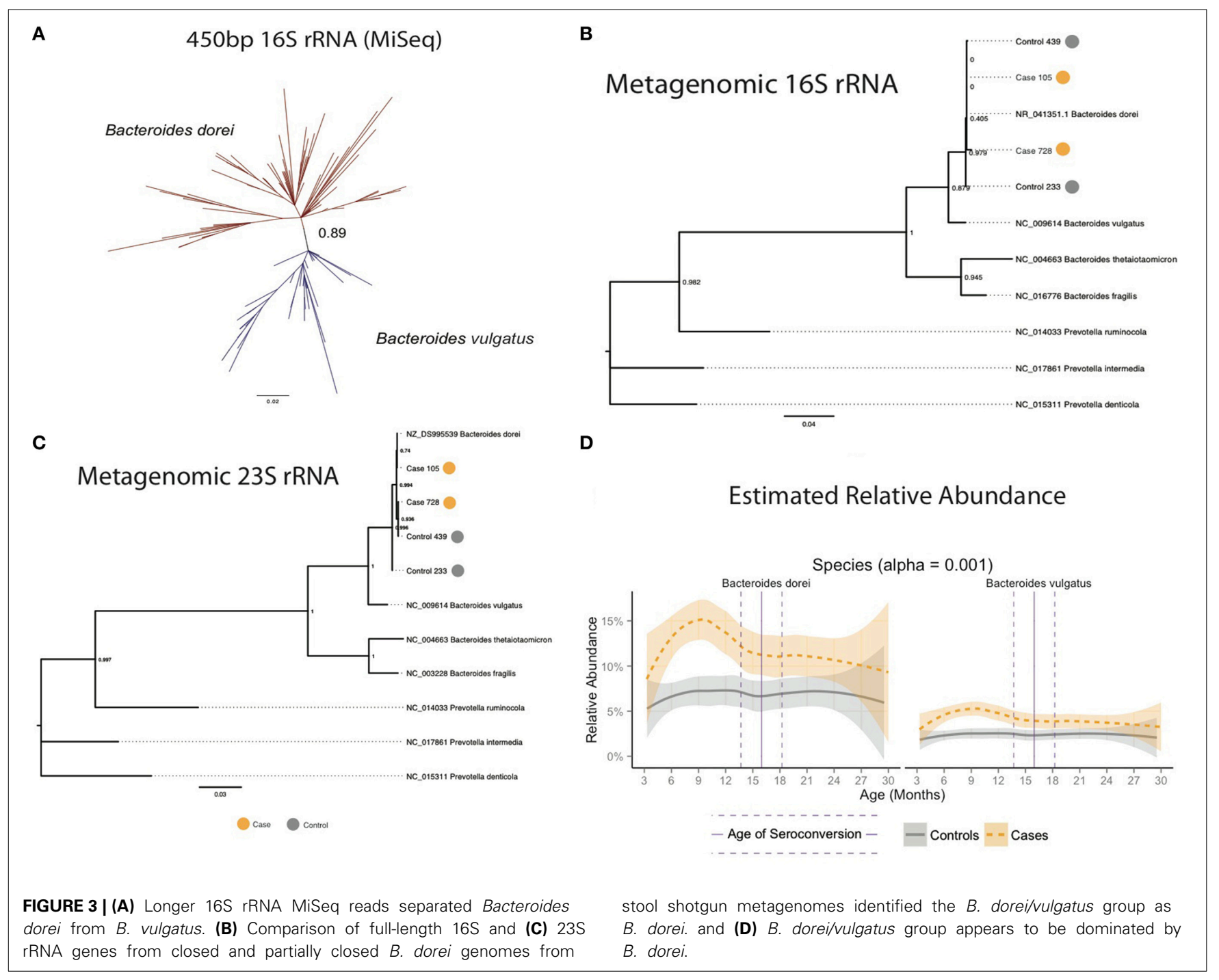

Fitted linear regressions were then used to estimate $B$. dorei and $B$. vulgatus relative abundance for the remaining samples based on HiSeq data (Figure 2D).

\section{DETERMINING TIMING OF Bacteroides dorei SPIKE}

To determine the specific timing of the B. dorei spike in cases, samples were binned into windows across age with average width of 98 days $(S E=0.33)$, two samples per individual and 98 samples per window $(S E=2.98)$. Windows with less than 20 cases and 20 controls were discarded. Downsampling was used to account for differences in subject sampling frequency by taking the median relative abundance for each taxon from multiple samples from the same individual. Bootstrapping was used to account for differences in sampling frequency between cases and controls averaging Mann-Whitney U p-values over 100 bootstraps within each window (Figure 3, Table S5).

\section{ESTIMATION OF ABSOLUTE Bacteroides dorei ABUNDANCE}

Sequencing of $16 \mathrm{~S}$ rRNA amplicons can only measure relative (proportion of the total) abundance. Therefore, the spike in relative abundance of $B$. dorei could be either a true spike in number of $B$. dorei cells or a decrease in the abundance of all other taxa. This leaves two possibilities, either B. dorei is associated with disease or the reduction in all of the other bacterial inhabitants are associated with disease but $B$. dorei remains. The total abundance of $B$. dorei was measured in order to distinguish the two possibilities. SYBR Green Quantitative PCR (qPCR) was used to estimate absolute abundance of the Bacteroides group using Bacteroides-specific primers that were shown to have high sensitivity and specificity (Liu et al., 2003). Twenty-eight samples taken from subjects between the age of 200 and 350 days were chosen based on (1) equal number of cases and controls; (2) good representation of the range of relative abundances; and (3) high DNA concentration $(20 \mathrm{ng} / \mathrm{uL})$. Amplification of a standard curve of PCR product generated from Bacteroides fragilis ATCC 25285 cDNA using the same primers in triplicate was used to calculate copy number in the stool DNA. Six samples were thrown out due to having high intra-triplicate variance. Relative abundance and absolute abundance were highly correlated using a Poisson linear regression ( $p=2.18 \times 10^{-35}$, see Supplementary Methods). 


\section{PREDICTING SEROCONVERSION BASED ON Bacteroides dorei RELATIVE ABUNDANCE}

The accuracy of a logistic regression model to measure the accuracy of predicting seroconversion based on the relative abundance of $B$. dorei was estimated using leave-one-subject-out crossvalidation (Table S5). Because of the possibility of the logistic regression classifying a subject as case or control could depend on recognition of that subject's microbiome rather than true differences between cases and controls, relative abundances were downsampled per subject and no sample from the same subject was used for training and testing. Cross-validation accuracy was measured using the Area Under the receiver operating Characteristic (AUC) with significance being measured using an analysis of variance with a chi-squared test (Table S5).

\section{AVAILABILITY OF SOURCE CODE AND DATA}

Source code used for the statistical analyses is available at https://github.com/triplett/dipp-microbiome-study. The 16S rRNA sequencing reads for this work are archived in NCBI BioProject PRJNA232731. The $B$. dorei genome sequenced in this study (from sample 728) is available under the NCBI accession CP009057. The Illumina HiSeq and MiSeq 16S sequences are available from NCBI's Short Read Archive under Study SRP043574. Table S10 contains a table of unrarefied 16S rRNA read counts at Phylum, Genus and Species ranks.

Analyses were performed using functions in the $\mathrm{R}$ programming language ( $\mathrm{R}$ Core Team, 2012) as well as the Phyloseq (McMurdie and Holmes, 2012) and ggplot2 (Wickham, 2009).

\section{RESULTS}

To examine the development of the gut microbiome in children at genetic risk for type 1 diabetes (T1D), DNA from 947 stool samples from 76 number children having the moderate (oddsratio $<5$ ) to high risk (odds-ratio 5-15) for T1D according to their HLA-DQB1 genotype, which is known to increase T1D risk, was sequenced. The HLA-DQ genotype is the most common high-risk genotype for T1D and confers a greater risk for future T1D than other high-risk alleles (Barrett et al., 2009). Twenty-nine subjects developed at least three persistent T1Drelative autoantibodies (mean age at diagnosis $=16.8$ months, $S E=2.03)$, which collectively confers $>95 \%$ risk of clinical T1D diagnosis. Those 29 subjects are considered "cases" for the purposes of this study. The remaining 47 subjects with no detectable T1D-related autoantibodies in serum were retroactively selected to serve as "controls" (Table S1). Genetic risk for T1D was balanced between case and control groups leaving the remaining risk up to environmental factors. To better control for environmental variables other than the gut microbiome including factors which are known to effect the microbiome such as mode of delivery (Dominguez-Bello et al., 2010), breast feeding (Cabrera-Rubio et al., 2012), and antibiotics (Dethlefsen et al., 2008; Jakobsson et al., 2010; Panda et al., 2014), subjects recruited for the retrospective, longitudinal DIPP study were examined for differences in these factors. None of these factors were significantly different between cases and controls ( $p>0.05$, see Supplementary Methods). No information on introduction of solid food was collected. However, months of exclusive breast feeding did not differ between cases and controls. All subjects were born in the Turku University Hospital in Turku, Finland allowing us to specifically examine differences in the gut microbiome compositions of individuals progressing to T1D-related autoimmunity vs. those not developing T1D-autoimmunity, while controlling for other environmental factors.

\section{GUT MICROBIOME DIFFERS BETWEEN CASES AND CONTROLS BEFORE SEROCONVERSION}

To determine the extent to which gut bacterial communities may differ between genetically at-risk subjects progressing toward T1D and those avoiding T1D progression, bacterial community $16 \mathrm{~S}$ rRNA genes were sequenced from 947 stool samples collected every 36 days $(S E=11)$ on average (Table S2) and were assigned sequences to "operational taxonomic units" (OTUs) in order to characterize the taxonomic composition of each sample's community. At the phylum level, the gut microbiomes were dominated by Bacteroidetes and Firmicutes followed by Actinobacteria and Proteobacteria (Figure 1A). At the genus level, these samples were high in relative abundance of Bacteroides(30-50\%) followed by moderate levels $(<10 \%)$ of Ruminococcus, Faecalibacterium, Blautia, Bifidobacterium, Veillonella, and Coprococcus (Figure 1B). Taxa below 1\% median relative abundance were filtered from the analysis as the abundance measurement of these taxa was unreliable in technical replicates (see Supplementary Methods). The remaining dominant taxa made up the vast the majority of the net microbial community consistent with previous studies on the human gut microbiomes of healthy and at-risk humans (Brown et al., 2011; Giongo et al., 2011; Kraal et al., 2014).

Gut microbiomes were compared between cases and controls by testing for differences in relative abundance across all samples and across age (see methods). All statistical tests were adjusted for false discovery rate, a common problem in studies with a highnumber of variables. Taxa below $1 \%$ were included in the FDR correction. Microbiome differences were only considered significant if their adjusted $p$-values fell below an alpha level of 0.001 . This approach is likely to make our approach conservative, while focusing on the high-abundance taxa more likely to have large effects on biological processes in the human gut.

Across all samples, the Gram-negative phylum Bacteroidetes was on average $6.7 \%$ higher in cases than controls (adjusted $p=2.17 \mathrm{e}-6)$ and the Gram-positive phyla Proteobacteria and Tenericutes were significantly higher in controls (adjusted $p=$ $1.59 \mathrm{e}-7$ and $7.20 \mathrm{e}-8$ respectively). Comparison of the gut microbiome across age at showed only Bacteroidetes and Firmicutes to be significantly different between cases and controls (Figure 1A, adjusted $p$-values $=1.35 \mathrm{e}-10,2.2 \mathrm{e}-5$, respectively). Further examination of the gut microbiome communities of cases vs. controls revealed that a single dominant genus Bacteroides was significantly higher in cases vs. controls using all samples at all time points with a median relative abundance of $44.4 \%$ in cases $(S E=$ $\left.5.5 \times 10^{-3}\right)$ and $30.7 \%\left(S E=10.2 \times 10^{-3}\right)$ in controls (adjusted $p=1.6 \mathrm{e} 0-7)$. Bacteroides and Coprococcus were found to vary significantly across age (Figure $2 \mathbf{B}$, adjusted $p$-values $=3.6 \mathrm{e}-11$, $8.5 \mathrm{e}-05$, respectively) although Coprococcus was only significantly 
higher in controls at a higher alpha considered in this study (adjusted $p=0.034$ ).

The Bacteroides genus was found to be dominated by two closely related species, Bacteroides dorei and Bacteroides vulgatus, which differed significantly between cases and controls across all samples, across age ( $p=1.78 \mathrm{e}-17$, Table S3) and within several windows prior to seroconversion (Figure 1C, $p<0.01$, Table S5). These two species were not distinguishable by their V4 16S rRNA region preventing further identification (see methods). Longer sequencing of the combined V3 and V4 regions in 93 samples differentiated the two species identifying $B$. dorei as the major constituent and both B. dorei and B. vulgatus (Figure 3C).

To provide further evidence of the identity of the $B$. dorei/vulgatus group as $B$. dorei, we performed shotgun metagenomic sequencing on four stool samples and succeeded in the closure of three genomes closely resembling $B$. dorei, two of which we reported previously (Leonard et al., 2014). These genomes had higher average nucleotide identity to $B$. dorei than $B$. vulgatus, as well as a beta-galactosidase gene which $B$. vulgatus lacks (Bakir et al., 2006). Assembly of the fourth genome did not result in genome closure. However, full-length $16 \mathrm{~S}$ and 23S rRNA genes mined from all four samples clustered at high confidence with $B$. dorei on a phylogenetic reconstruction (Figures $3 \mathbf{A}, \mathbf{B}$ ). Relative abundance of $B$. dorei in these metagenomes closely matched that measured by $16 \mathrm{~S}$ rRNA sequences $\left(R^{2}=0.97\right.$, Table S9).

$B$. dorei made up roughly $75 \%$ of the $B$. dorei/vulgatus group (Figure 3D, see methods) and was significantly higher in cases across age using the logistic regression approach $(p<0.001)$. $B$. dore $i$ was 5 times more abundant in cases than controls across all samples and reached up to 100 times higher in cases $(17.3 \%$ relative abundance, $S E=0.02)$ versus controls $(0.18 \%, S E=$ 0.01 ) within a 78 -day window at 7.5 month of age, or 9.2 months prior to the average age of first T1D autoantibody $(p=0.005$, Figure 4, Table S5).

High-throughput sequencing is limited by its ability to only measure relative as opposed to absolute abundance. As a result, an apparent increase in the relative abundance of a taxon could be the result of either an actual increase in absolute abundance of that taxon or a decrease in all remaining taxa. To overcome this limitation, quantitative PCR was done on 22 stool DNA samples using universal Bacteroides-specific primers. The relative abundance of Bacteroides was highly correlated with absolute abundance (see Supplementary Methods). Therefore, this increase in $B$. dorei is likely due to an increase in actual number of cells, not a decrease in all other taxa.

To test whether the $B$. dorei can be used as a biomarker to predict future autoimmunity and type-1 diabetes, we tested efficacy of classification of case/control status based on B. dorei abundance using cross-validation, which estimates a predictor's accuracy on real-world data. Cross-validation was performed using the sliding window across age and accuracy was found to peak in samples taken between 6.1 and 12.1 months of age, between 12 and 15 months before the first detection of T1D autoimmunity (Figure 5, Table S5). Accuracy measured by the Area Under the Curve (AUC) to be 0.69 ( $S E=0.007)$ which was significantly higher than chance ( $p=0.019$, Figures 4,5 , Table S5) using data downsampled by subject in order to avoid overfitting to subjects' microbiomes. However, a true, blind leave-out dataset is required in order to measure the accuracy of microbiome-based prediction of autoimmunity on real-world data (Babyak, 2004).

\section{DISCUSSION}

In this cohort, high levels of Bacteroides dorei in the gut are associated with future autoimmunity for T1D. Bacteroides dorei is a recently discovered species isolated from the human gut

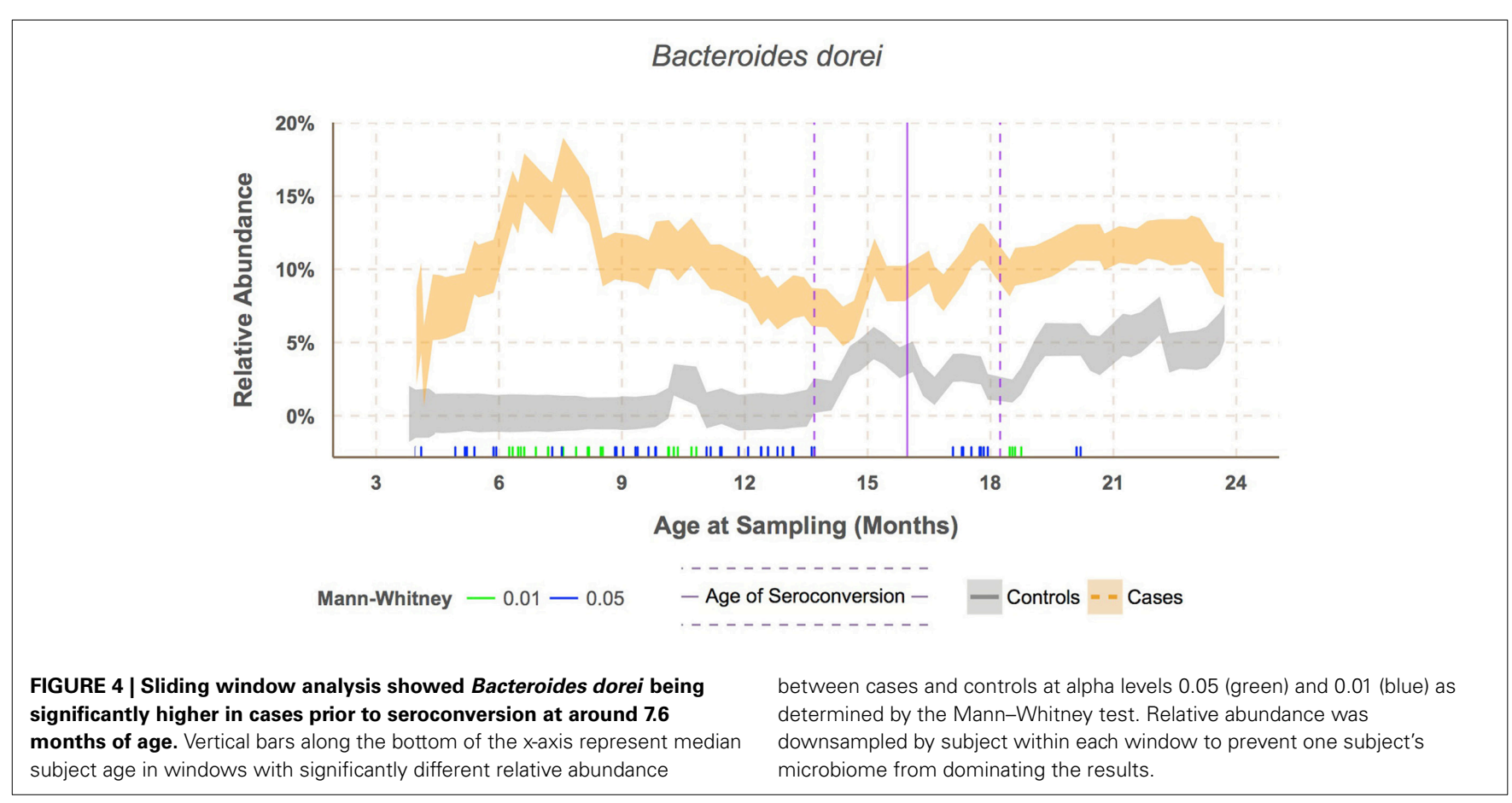




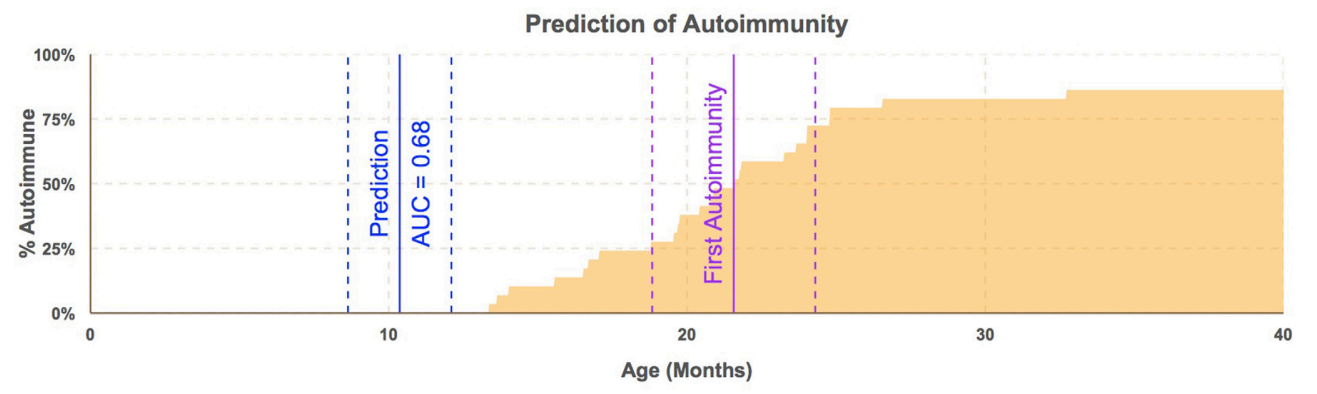

FIGURE 5 | Bacteroides dorei/vulgatus predicts autoimmunity before appearance of any single autoantibody. Timing of prediction of autoimmunity. Blue line represents median age (11 months) of subjects in windows of samples taken from subjects aged 6.1-12.1 months with standard error lines (dashed). The purple line represents median age of the appearance of the first autoantibody with standard error lines (dashed). The proportion of cases who have seroconverted is plotted across time (orange area). Estimation of the efficacy of $B$. dorei to serve as a predictor of future autoimmunity was significantly better than chance within this window (AUC $=0.68, S E=0.007$ ) before the appearance of any single autoantibody.
(Bakir et al., 2006) and may be mistaken with B. vulgatus due to their taxonomic similarity (Bakir et al., 2006). However, whole genome analysis shows that the two species are sufficiently different to warrant separate species designations. Nevertheless, the two species are very similar and have not yet been distinguished from each other physiologically apart from beta-galactosidase activity in B. dorei (Bakir et al., 2006).

Higher levels of Bacteroides in cases were observed previously in a rat model for T1D as well as in human cohorts (Roesch et al., 2009b; Brown et al., 2011; Giongo et al., 2011). However, this work advances our knowledge in three ways. First, it is the largest human T1D cohort examined to date and is a cohort derived from a single city with all children born in the same hospital. Second, a frequent sampling of the subjects occurred from about 6 months after birth until 3 years of age, which provides the ability to observe the development of the microbiome across age and identify potential associations. And third, the analyses performed here suggest a role for a single, dominant bacterial species associated with T1D autoimmunity.

Increased abundance of Bacteroides species including $B$. dorei and $B$. vulgatus have been implicated in inflammation in several gut diseases such as ulcerative colitis (UC), irritable bowel disease (IBD), and celiac disease (CD) (Breeling et al., 1988; Rath et al., 1999; Fujita et al., 2002; Sanchez et al., 2010, 2011; Sato et al., 2010; Schippa et al., 2010; Bloom et al., 2011). A recent report showed that $B$. vulgatus was found in duodenal biopsy specimens of $70 \%$ of subjects ( 14 of 20 ) with untreated $C D$ versus $0 \%$ of subjects $(0$ of 12$)$ treated with a gluten-free diet for at least two years (Schippa et al., 2010).

While it has not been shown whether B. vulgatus or B. dorei are causative rather than correlated with disease, B. vulgatus induces colitis in rats, mice, and guinea pigs (Salyers et al., 1977; Fujita et al., 2002; Sanchez et al., 2011). Strains of B. vulgatus associated with human UC have a rougher surface and higher cell adherence rates than $B$. vulgatus strains derived from non-UC patients (Schippa et al., 2010). B. vulgatus is not thought to directly cause IBD but may prevent its remission (Fujita et al., 2002) and is also known as an opportunistic pathogen causing intra-abdominal infections (Rath et al., 1999). Infants with high-risk HLA genotypes for T1D and CD had higher prevalence of $B$. vulgatus than infants at low genetic risk (Sanchez et al., 2011). B. vulgatus was more common in CD patients, and B. dorei was more common in patients with active CD (Sanchez et al., 2010).

The cause of increased $B$. dorei in these subjects is not known. Bacteroides are observed in infants after the first introduction of solid food (Nwaru et al., 2010). Bacteroides colonization of the human gut at this early stage of life is important given their role in the digestion of complex carbohydrates (Salyers et al., 1977). At the time of the infant period for the current study participants, the national health care system in Finland recommended that parents should introduce solid food between the age of 4 and 6 months (Simell and Aula, 1997; Hasunen et al., 2004). Thus, the appearance of a large proportion of Bacteroides in these children at 6 months of age appears to be associated with the introduction of solid food.

Bacteroides possess high numbers of antibiotics resistance genes. It is possible that the increase in antibiotic usage, especially in developed countries, has led to an overall increase in Bacteroides across entire populations and in their environment. This might explain the recent increase in T1D prevalence as well as country-by-country differences. This study lacks sufficient data to estimate the effect of antibiotics on Bacteroides abundance in the gut and risk for autoimmunity and T1D. Larger cohorts with an antibiotic control group are needed to investigate this potential link.

Whether B. dorei plays a role in causing T1D autoimmunity in these children or is simply a consequence of emerging autoimmunity, overall health, or yet another environmental factor is unknown. If $B$. dorei is involved in he development of autoimmunity, likely routes include disruption of the epithelial layer and/or manipulation of immune system development. If this is the case, can high levels of $B$. dorei gut colonization be prevented? Bacteroides strains are resistant to antibiotics commonly used in pediatrics such as amoxicillin. The $B$. dorei genome sequenced from these stool samples possesses over 50 genes annotated as antibiotic resistance determinants (Leonard et al., 2014). However, two antibiotics, doripenem and tigecycline, are both 
injected to treat intra-abdominal infections caused by $B$. dorei (Brook et al., 2013). Whether they would be useful in reducing $B$. dorei gut colonization through oral administration is unclear. Alternatively, Bifidobacterium strains decrease the population of B. vulgatus in the gut (Shiba et al., 2003) and ameliorate gut inflammation by reducing $B$. vulgatus gut populations of gnotobiotic mice (Setoyama et al., 2003).

Another possibility is that a diet change could reduce the population size of $B$. dorei. Previous work has shown that the proportion of children with $B$. vulgatus or $B$. dorei in the gut declines when a gluten-free diet is introduced after diagnosis of celiac disease (Sanchez et al., 2010). In addition, the gut microbiome of children at high risk for celiac disease are more likely to include $B$. dorei or $B$. vulgatus than the microbiomes of low risk children (Sanchez et al., 2011). In addition, high protein and fat diets are correlated with high Bacteroides populations (Wu et al., 2011).

Results presented here show that the microbiome differs within a window of several months preceding the appearance of the first anti-islet autoantibodies. As such, even if $B$. dorei is not the cause of T1D autoimmunity, its abundance in the gut may be useful as a diagnostic in high-risk groups, such as first-degree relatives, at 6-8 months of age, prior to the appearance of a single autoantibody. At present, infants are not commonly screened for their HLA genotype at birth. Hence, a child's high genetic risk for T1D is only easily determined by the parents if the child has a first degree relative with the disease. As our knowledge of microbiome associations with T1D expands, gut microbiome testing through non-invasive stool analysis might be recommended in the future for infants with a first degree relative affected by T1D.

Type 1 diabetes is a complex disease and is thought to be a cluster of similar diseases with different etiologies (Knip et al., 2005). Studying multiple forms of a disease as a whole may mask differences specific to a single sub-type and fail to discover novel associations. For example, in the BABYDIET study (Endesfelder et al., 2014), which examined the development microbiomes of children from across Germany, no associations were found. In this study, all subjects were born in the same hospital in Turku, Finland and remained in Turku and surrounding areas for the study's duration. As a result, many factors are very similar for all Turku children across this cohort such as climate, health standards and practices, quality of drinking water, exposure to environmental pollutants, diet, and vitamin $\mathrm{D}$ levels from sun exposure. Controlling of all of these environmental factors may increase the likelihood that subjects who develop type-1 diabetes have the same form of the disease with the same etiology. Thus, studying large cohorts from one city or small geographic region is particularly valuable.

Bacteroides dorei may or may not be associated with T1D autoimmunity in other disease forms, which are more prevalent in other locations. Alternatively, and perhaps more likely, the key to understanding the role of the microbiome in T1D may not be the identification of a specific bacterium, but a set of specific bacterial genes related to gut health. It just so happens, that in this one city, Turku, Finland, those functions may largely be associated with one bacterial species.

\section{ACKNOWLEDGMENTS}

This research was generously supported by grants from JDRF, the Juvenile Diabetes Research Foundation (www.jdrf.org) (Project 17-2011-266 to Eric W. Triplett). The DIPP study was supported by grants from Juvenile Diabetes Research Foundation (Projects 4-1998-274, 4-1999-731, 4-2001-435, and 36-2008919), European Union (Project TORNADO, FP7-KBBE-222720), Academy of Finland (Centre of Excellence in Molecular Systems Immunology and Physiology Research, 2012-2017, Decision No. 250114), Emil Aaltonen Foundation, Jalmari and Rauha Ahokas Foundation, Signe and Ane Gyllenberg Foundation, Sigrid Juselius Foundation, Päivikki and Sakari Sohlberg Foundation and Special Research Funds for University Hospitals in Finland. The funders had no role in study design, data collection and analysis, decision to publish, or preparation of the manuscript.

\section{SUPPLEMENTARY MATERIAL}

The Supplementary Material for this article can be found online at: http://www.frontiersin.org/journal/10.3389/fmicb. 2014.00678/abstract

\section{REFERENCES}

Atkinson, M. A., Eisenbarth, G. S., and Michels, A. W. (2014). Type 1 diabetes. Lancet 383, 69-82. doi: 10.1016/S0140-6736(13)60591-7

Babyak, M. A. (2004). What you see may not be what you get: a brief, nontechnical introduction to overfitting in regression-type models. Psychosom. Med. 66, 411-421. doi: 10.1097/01.psy.0000127692.23278.a9

Bach, J. F. (2002). The effect of infections on susceptibility to autoimmune and allergic diseases. N. Engl. J. Med. 347, 911-920. doi: 10.1056/NEJMra 020100

Barrett, J. C., Clayton, D. G., Concannon, P., Akolkar, B., Cooper, J. D., Erlich, H. A., et al. (2009). Genome-wide association study and meta-analysis find that over 40 loci affect risk of type 1 diabetes. Nat. Genet. 41, 703-707. doi: 10.1038/ng.381

Bakir, M. A., Sakamoto, M., Kitahara, M., Matsumoto, M., and Benno, Y. (2006). Bacteroides dorei sp. nov., isolated from human faeces. Int. J. Syst. Evol. 56, 1639-1643. doi: 10.1099/ijs.0.64257-0

Benjamini, Y., and Hochberg, Y. (1995). Controlling the false discovery rate: a practical and powerful approach to multiple testing. J. R. Stat. Soc. B 57, 289-300.

Bloom, S. M., Bijanki, V. N., Nava, G. M., Sun, L., Malvin, N. P., Donemeyer, D. L., et al. (2011). Commensal Bacteroides species induce colitis in host-genotypespecific fashion in a mouse model of inflammatory bowel disease. Cell Host Microbe 9, 390-403. doi: 10.1016/j.chom.2011.04.009

Breeling, J. L., Onderdonk, A. B., Cisneros, R. L., and Kasper, D. L. (1988) Bacteroides vulgatus outer membrane antigens associated with carrageenaninduced colitis in guinea pigs. Infect. Immun. 56, 1754-1759.

Brook, I., Wexler, H. M., and Goldstein, E. J. (2013). Antianaerobic antimicrobials: spectrum and susceptibility testing. Clin. Microbiol. Rev. 26, 526-546. doi: 10.1128/CMR.00086-12

Brown, C. T., Davis-Richardson, A. G., Giongo, A., Gano, K. A., Crabb, D. B., Mukherjee, N., et al. (2011). Gut microbiome metagenomics analysis suggests a functional model for the development of autoimmunity for type 1 diabetes. PLoS ONE 6:e25792. doi: 10.1371/journal.pone.0025792

Brugman, S., Klatter, F. A., Visser, J. T., Wildeboer-Veloo, A. C., Harmsen, H. J., Rozing, J., et al. (2006). Antibiotic treatment partially protects against type 1 diabetes in the Bio-Breeding diabetes-prone rat. Is the gut flora involved in the development of type 1 diabetes? Diabetologia 49, 2105-2108. doi: 10.1007/s00125-006-0334-0

Cabrera-Rubio, R., Collado, M. C., Laitinen, K., Salminen, S., Isolauri, E., and Mira, A. (2012). The human milk microbiome changes over lactation and is shaped by maternal weight and mode of delivery. Am. J. Clin. Nutr. 96, 544-551. doi: 10.3945/ajcn.112.037382 
Caporaso, J. G., Kuczynski, J., Stombaugh, J., Bittinger, K., Bushman, F. D., Costello, E. K., et al. (2010). QIIME allows analysis of high-throughput community sequencing data. Nat. Methods 7, 335-336. doi: 10.1038/nmeth.f.303

Caporaso, J. G., Lauber, C. L., Walters, W. A., Berg-Lyons, D., Lozupone, C. A., Turnbaugh, P. J., et al. (2011). Global patterns of $16 \mathrm{~S}$ rRNA diversity at a depth of millions of sequences per sample. Proc. Natl. Acad. Sci. U.S.A. 108, 4516-4522. doi: 10.1073/pnas.1000080107

de Goffau, M. C., Luopajarvi, K., Knip, M., Ilonen, J., Ruohtula, T., Harkonen, T., et al. (2013). Fecal microbiota composition differs between children with $\beta$-cell autoimmunity and those without. Diabetes 62, 1238-1244. doi: $10.2337 / \mathrm{db} 12$ 0526

DeSantis, T. Z., Hugenholtz, P., Larsen, N., Rojas, M., Brodie, E. L., Keller, K., et al. (2006). Greengenes, a chimera-checked 16S rRNA gene database and workbench compatible with ARB. Appl. Environ. Microbiol. 72, 5069-5072. doi: 10.1128/AEM.03006-05

Dethlefsen, L., Huse, S., Sogin, M. L., and Relman, D. A. (2008). The pervasive effects of an antibiotic on the human gut microbiota, as revealed by deep 16S rRNA sequencing. PLoS Biol. 6:e280. doi: 10.1371/journal.pbio.0060280

Dominguez-Bello, M. G., Costello, E. K., Contreras, M., Magris, M., Hidalgo, G., Fierer, N., et al. (2010). Delivery mode shapes the acquisition and structure of the initial microbiota across multiple body habitats in newborns. Proc. Natl. Acad. Sci. U.S.A. 107, 11971-11975. doi: 10.1073/pnas.1002601107

Edgar, R. C. (2004). MUSCLE: multiple sequence alignment with high accuracy and high throughput. Nucleic Acids Res. 32, 1792-1797. doi: 10.1093/nar/ gkh340

Edgar, R. C. (2010). Search and clustering orders of magnitude faster than BLAST. Bioinformatics 28, 2460-2461. doi: 10.1093/bioinformatics/btq461

Endesfelder, D., zu Castell, W., Ardissone, A., Davis-Richardson, A. G., Achenbach, P., Hagen, M., et al. (2014). Compromised gut microbiota networks in children with anti-islet cell autoimmunity. Diabetes 63, 2006-2014. doi: 10.2337/ db13-1676

Fagen, J. R., Giongo, A., Brown, C. T., Davis-Richardson, A. G., Gano, K. A., and Triplett, E. W. (2012). Characterization of the relative abundance of the citrus pathogen $\mathrm{Ca}$. Liberibacter asiaticus in the microbiome of its insect vector, Diaphorina citri, using high throughput 16S rRNA sequencing. Open Microbiol. J. 6, 29-33. doi: 10.2174/1874285801206010029

Fujita, H., Eishi, Y., Ishige, I., Saitoh, K., Takizawa, T., Arima, T., et al. (2002). Quantitative analysis of bacterial DNA from Mycobacteria spp., Bacteroides vulgatus, and Escherichia coli in tissue samples from patients with inflammatory bowel diseases. J. Gastroenterol. 37, 509-516. doi: 10.1007/s005350200079

Giongo, A., Gano, K. A., Crabb, D. B., Mukherjee, N., Novelo, L. L., Casella, G., et al. (2011). Toward defining the autoimmune microbiome for type 1 diabetes. ISME J. 5, 82-91. doi: 10.1038/ismej.2010.92

Guindon, S., Lethiec, F., Duroux, P., and Gascuel, O. (2005). PHYML Online-a web server for fast maximum likelihood-based phylogenetic inference. Nucleic Acids Res. 33, W557-W559. doi: 10.1093/nar/ gki352

Hasunen, K., Kalavainen, M., Keinonen, H., Lagstrom, H., Lyytikainen, A., Nurttila, A., et al. (2004). The Child, Family and Food. Nutrition Recommendations for Infants and Young Children as Well as Pregnant and Breastfeeding Mothers. Helsinki: Publications of the Finnish Ministry of Social Affairs and Health.

Hummel, S., Pflüger, M., Hummel, M., Bonifacio, E., and Ziegler, A. G. (2011). Primary dietary intervention study to reduce the risk of islet autoimmunity in children at increased risk for type 1 diabetes: the BABYDIET study. Diabetes Care 34, 1301-1305. doi: 10.2337/dc10-2456

Jakobsson, H. E., Jernberg, C., Andersson, A. F., Sjölund-Karlsson, M., Jansson, J. K., and Engstrand, L. (2010). Short-term antibiotic treatment has differing long-term impacts on the human throat and gut microbiome. PLoS ONE 5:e9836. doi: 10.1371/journal.pone.0009836

Joshi, N. (2013). Sickle Windowed Adaptive Trimming for Fastq Files Using Quality. Available online at: https://github.com/najoshi/sickle (Accessed January 13, 2014).

Karvonen, M., Tuomilehto, J., Libman, I., and LaPorte, R. (1993). A review of the recent epidemiological data on the worldwide incidence of type 1 (insulin-dependent) diabetes mellitus. World Health Organization DIAMOND Project Group. Diabetologia 36, 883-892. doi: 10.1007/BF02374468

King, C., and Sarvetnick, N. (2011). The incidence of type-1 diabetes in NOD mice is modulated by restricted flora not germ-free conditions. PLOS ONE 6:e17049. doi: 10.1371/journal.pone.0017049
Knip, M., Veijola, R., Virtanen, S. M., Hyöty, H., Vaarala, O., and Åkerblom, H. K. (2005). Environmental triggers and determinants of type 1 diabetes. Diabetes 54, S125-S136. doi: 10.2337/diabetes.54.suppl_2.S125

Kraal, L., Abubucker, A., Kota, K., Fischbach, M. A., and Mitreva, M. (2014). The prevalence of species and strains in the human microbiome: a resource for experimental efforts. PLOS ONE 9:e97279. doi: 10.1371/journal.pone.0097279

Lagesen, K., Hallin, P., Rødland, E. A., Stærfeldt, H. H., Rognes, T., and Ussery, D. W. (2007). RNAmmer: consistent and rapid annotation of ribosomal RNA genes. Nucleic Acids Res. 35, 3100-3108. doi: 10.1093/nar/gkm160

Leonard, M. T., Davis-Richardson, A. G., Ardissone, A. N., Kemppainen, K., Drew, J. C., Ilonen, J., et al. (2014). The methylome of the gut microbiome: disparate Dam methylation patterns in intestinal Bacteroides dorei of children. Front. Microbiol. 5:361. doi: 10.3389/fmicb.2014.00361

Liu, Z., Lozupone, C., Hamady, M., Bushman, F. D., and Knight, R. (2007). Short pyrosequencing reads suffice for accurate microbial community analysis. Nucleic Acids Res. 35, e120. doi: 10.1093/nar/gkm541

Liu, C., Song, Y., McTeague, M., Vu, A. W., Wexler, H., and Finegold, S. M. (2003). Rapid identification of the species of the Bacteroides fragilis group by multiplex PCR assays using group - and species - specific primers. FEMS Microbiol. Lett. 222, 9-16. doi: 10.1016/S0378-1097(03)00296-9

Masella, A. P., Bartram, A. K., Truszkowski, J. M., Brown, D. G., and Neufeld, J. D. (2012). PANDAseq: paired-end assembler for illumina sequences. BMC Bioinformatics13:31. doi: 10.1186/1471-2105-13-31

McMurdie, P. J., and Holmes, S. (2012). Phyloseq: a bioconductor package for handling and analysis of high-throughput phylogenetic sequence data. Pac. Symp. Biocomput. 17, 235-246. doi: 10.1142/9789814366496_0023

Muyzer, G., Hottenträger, S., Teske, A., and Wawer, C. (1996). Denaturing gradient gel electrophoresis of PCR-amplified $16 \mathrm{~S}$ rDNA - a new molecular approach to analyse the genetic diversity of mixed microbial communities. Mol. Microbial. Ecol. Manual 3, 1-23.

Nejentsev, S., Sjoroos, M., Soukka, T., Knip, M., Simell, O., Lövgren, T., et al. (1999). Population-based genetic screening for the estimation of Type 1 diabetes mellitus risk in Finland: selective genotyping of markers in the HLADQB1, HLA-DQA1 and HLA-DRB1 loci. Diabet. Med. 16, 985-992. doi: 10.1046/j.1464-5491.1999.00186.x

Nwaru, B. I., Erkkola, M., Ahonen, S., Kaila, M., Haapala, A. M., KronbergKippilä, C., et al. (2010). Age at the introduction of solid foods during the first year and allergic sensitization at age 5 years. Pediatrics 125, 50-59. doi: 10.1542/peds.2009-0813

Panda, S., Guarner, F., and Manichanh, C. (2014). Structure and functions of the gut microbiome. Endocr. Metab. Immune. Disord. Drug Targets 14, 290-299. doi: 10.2174/1871530314666140714120744

Parikka, V., Näntö-Salonen, K., Saarinen, M., Simell, T., Ilonen, J., Hyöty, H., et al. (2012). Early seroconversion and rapidly increasing autoantibody concentrations predict prepubertal manifestation of type 1 diabetes in children at genetic risk. Diabetologia 55, 1926-1936. doi: 10.1007/s00125-012-2523-3

Pedersen, P. M., Marmolin, E. S., and Justesen, U. S. (2013). Species differentiation of Bacteroides dorei from Bacteroides vulgatus and Bacteroides ovatus from Bacteroides xylanisolvens - back to basics. Anaerobe 24, 1-3. doi: 10.1016/j.anaerobe.2013.08.004

Rath, H. C., Wilson, K. H., and Sartor, R. B. (1999). Differential induction of colitis and gastritis in HLA-B27 transgenic rats selectively colonized with Bacteroides vulgatus or Escherichia coli. Infect. Immun. 67, 2969-2974.

R Core Team. (2012). R: A Language and Environment for Statistical Computing. Vienna: The R Foundation for Statistical Computing.

Roesch, L. F. W., Casella, G., Simell, O., Krischer, J., Wasserfall, C. H., Schatz, D., et al. (2009a). Influence of sample storage on bacterial community diversity in fecal samples. Open Microbiol. J. 3, 40-46. doi: 10.2174/1874285800903010040

Roesch, L. F., Lorca, G. L., Casella, G., Giongo, A., Naranjo, A., Pionzio, A. M., et al. (2009b). Culture-independent identification of gut bacteria correlated with the onset of diabetes in a rat model. ISME J. 3, 536-548 doi: 10.1038/ismej.2009.5

Salyers, A. A., Vercellotti, J. R., West, S. E., and Wilkins, T. D. (1977). Fermentation of mucin and plant polysaccharides by strains of Bacteroides from the human colon. Appl. Environ. Microbiol. 33, 319-322.

Sanchez, E., Donat, E., Ribes-Koninckx, C., Calabuig, M., and Sanz, Y. (2010). Intestinal Bacteroides species associated with coeliac disease. J. Clin. Pathol. 63, 1105-1111. doi: 10.1136/jcp.2010.076950

Sanchez, E., De Palma, G., Capilla, A., Nova, E., Pozo, T., Castillejo, G., et al. (2011). Influence of environmental and genetic factors linked to celiac disease 
risk on infant gut colonization by Bacteroides species. Appl. Environ. Microbiol. 77, 5316-5323. doi: 10.1128/AEM.00365-11

Sato, K., Kumita, W., Ode, T., Ichinose, S., Ando, A., Fujiyama, Y., et al. (2010). OmpA variants affecting the adherence of ulcerative colitis-derived Bacteroides vulgatus. J. Med. Dent. Sci. 57, 55-64.

Schippa, S., Lebba, V., Barbato, M., Di Nardo, G., and Totino, V. (2010). A distinctive 'microbial signature' in celiac pediatric patients. BMC Microbiol. 10:175. doi: 10.1186/1471-2180-10-175

Schmid, S., Buuck, D., Knopff, A., Bonifacio, E., and Ziegler, A. G. (2004). BABYDIET, a feasibility study to prevent the appearance of islet autoantibodies in relatives of patients with Type 1 diabetes by delaying exposure to gluten. Diabetologia 47, 1130-1131. doi: 10.1007/s00125-004-1420-9

Schwartz, R. F., Neu, J., Schatz, D., Atkinson, M. A., and Wasserfall, C. (2007). Comment on: Brugman S et al. (2006) Antibiotic treatment partially protects against type 1 diabetes in the Bio-Breeding diabetes-prone rat. Is the gut flora involved in the development of type 1 diabetes? Diabetologia 49: 2105-2108. Diabetologia 50, 220-221. doi: 10.1007/s00125-006-0526-7

Setoyama, H., Imaoka, A., Ishikawa, H., and Umesaki, Y. (2003). Prevention of gut inflammation by Bifidobacterium in dextran sulfate-treated gnotobiotic mice associated with Bacteroides strains isolated from ulcerative colitis patients. Microbes Infect. 5, 115-122. doi: 10.1016/S1286-4579(02)00080-1

Shiba, T., Aiba, Y., Ishikawa, H., Ushiyama, A., Takagi, A., Mine, T., et al. (2003). The suppressive effect of bifidobacteria on Bacteroides vulgatus, a putative pathogenic microbe in inflammatory bowel disease. Microbiol. Immunol. 47, 371-378. doi: 10.1111/j.1348-0421.2003.tb03368.x

Simell, O., and Aula, P. (1997). Neuvolakirja. London: Orion. ISBN 952983909X.

TEDDY Study Group. (2008). The environmental determinants of diabetes in the young (TEDDY) study. Ann. N.Y. Acad. Sci. 1150, 1. doi: 10.1196/annals.1447.062

Valladares, R., Sankar, D., Li, N., Williams, E., Lai, K. K., Abdelgeliel, A. S., et al. (2010). Lactobacillus johnsonii N6.2 mitigates the development of type 1 diabetes in BB-DP rats. PLoS ONE 5:e10507. doi: 10.1371/journal.pone.0010507
Wen, L., Ley, R. E., Vochkov, P. Y., Stranges, P. B., Avanesyan, L., Stonebraker, A. C., et al. (2008). Innate immunity and intestinal microbiota in the development of Type 1 diabetes. Nature 455, 1109-1113. doi: 10.1038/nature 07336

Wickham, H. (2009). ggplot2: Elegant Graphics for Data Analysis. New York, NY: Springer.

Wu, G. D., Chen, J., Hoffmann, C., Bittinger, K., Chen, Y.-Y., Keilbaugh, S. A., et al. (2011). Linking long-term dietary patterns with gut microbial enterotypes. Science 334, 105-108. doi: 10.1126/science. 1208344

Conflict of Interest Statement: The authors declare that the research was conducted in the absence of any commercial or financial relationships that could be construed as a potential conflict of interest.

Received: 11 September 2014; accepted: 19 November 2014; published online: 10 December 2014.

Citation: Davis-Richardson AG, Ardissone AN, Dias R, Simell V, Leonard MT, Kemppainen KM, Drew JC, Schatz D, Atkinson MA, Kolaczkowski B, Ilonen J, Knip M, Toppari J, Nurminen N, Hyöty H, Veijola R, Simell T, Mykkänen J, Simell O and Triplett EW (2014) Bacteroides dorei dominates gut microbiome prior to autoimmunity in Finnish children at high risk for type 1 diabetes. Front. Microbiol. 5:678. doi: 10.3389/fmicb.2014.00678

This article was submitted to Evolutionary and Genomic Microbiology, a section of the journal Frontiers in Microbiology.

Copyright $\odot 2014$ Davis-Richardson, Ardissone, Dias, Simell, Leonard, Kemppainen, Drew, Schatz, Atkinson, Kolaczkowski, Ilonen, Knip, Toppari, Nurminen, Hyöty, Veijola, Simell, Mykkänen, Simell and Triplett. This is an open-access article distributed under the terms of the Creative Commons Attribution License (CC BY). The use, distribution or reproduction in other forums is permitted, provided the original author(s) or licensor are credited and that the original publication in this journal is cited, in accordance with accepted academic practice. No use, distribution or reproduction is permitted which does not comply with these terms. 